\title{
Analysis of Line Contact Elastohydrodynamic Lubrication with the Particles under Rough Contact Surface
}

\author{
Keying Chen $\mathbb{D},{ }^{1,2}$ Liangcai Zeng $\mathbb{D}^{1,2}$ Juan Chen $\mathbb{D}^{1,2}$ and Xianzhong Ding $\mathbb{D}^{1,2}$ \\ ${ }^{1}$ Key Laboratory of Metallurgical Equipment and Control Technology, Wuhan University of Science and Technology, \\ Ministry of Education, Wuhan, Hubei 430081, China \\ ${ }^{2}$ Hubei Key Laboratory of Mechanical Transmission and Manufacturing Engineering, \\ Wuhan University of Science and Technology, Wuhan, Hubei 430081, China \\ Correspondence should be addressed to Liangcai Zeng; zlc0983@hotmail.com
}

Received 14 June 2019; Accepted 13 January 2020; Published 28 March 2020

Academic Editor: Marco Rossi

Copyright $\odot 2020$ Keying Chen et al. This is an open access article distributed under the Creative Commons Attribution License, which permits unrestricted use, distribution, and reproduction in any medium, provided the original work is properly cited.

\begin{abstract}
A numerical solution for line contact elastohydrodynamic lubrication (EHL) occurring on the rough surface of heterogeneous materials with a group of particles is presented in this study. The film thickness disturbance caused by particles and roughness is considered into the solution system, and the film pressure between the contact gap generated by the particles and the surface roughness is obtained through a unified Reynold equation system. The inclusions buried in the matrix are made equivalent to areas with the same material as that of the matrix through Eshelby's equivalent inclusion method and the roughness is characterized by related functions. The results present the effects of different rough topographies combined with the related parameters of the particles on the EHL performance, and the minimum film thickness distribution under different loads, running speeds, and initial viscosities are also investigated. The results show that the roughness morphology and the particles can affect the behavior of the EHL, the traction force on a square rough surface is smaller, and the soft particles have more advantages for improving the EHL performance.
\end{abstract}

\section{Introduction}

The improvement in working load of mechanical components is a major challenge for the development of sophisticated equipment. Elastohydrodynamic lubrication (EHL) is a common theory to explain the problem of surface deformation and rheological characteristics under working conditions. Most studies of EHL with inhomogeneous materials are usually carried out on smooth surfaces. However, in reality, the contact surfaces of the mechanical components are usually rough, and surface roughness is one of the important factors that cause failure of the lubrication film in EHL contact. In addition, the inhomogeneity can change the elastic field of the matrix, and then the film thickness and pressure of the EHL will be affected by the particles indirectly. Therefore, it is meaningful to study EHL with particles on rough contact surfaces to observe the behavior of the lubrication process and improve the lubrication environment.
The contact components with inhomogeneities have been investigated by many researchers. The heterogeneous particles distributed in the matrix can be described as a domain that has the same material properties as the matrix but with eigenstrains (proposed by Mura) [1] through the equivalent inclusion method (EIM) proposed by Eshelby [2]. Then, to solve the contact problem of inhomogeneous materials, a semianalytic solution for inhomogeneous inclusions with arbitrary shape in an isotropic half space under contact loading through the EIM was proposed by Zhou et al. and is widely used [3]. The contact pressure and the subsurface stress field for the contact problem of anisotropic elastic inhomogeneities with ellipsoidal shape were analyzed by Koumi et al. [4]. Recently, Zhou et al. presented numerical solution for contact problems of inhomogeneities with arbitrary shape and different material properties under rough and smooth surfaces based on the EIM [5]. Zuccaro et al. obtained an analytical solution of the elastic field of 2D inclusions whose boundary is approximated by a closed polygon [6]. 
It is well known that the EHL film thickness is very thin, which makes the film sensitive to the deformation and morphology of the contact surface. However, the presence of inhomogeneities such as coatings, fibers, and particles can change the internal structure and material properties of the contact components. Thus, inhomogeneities have attracted considerable attention in the study of EHL over the past decade. Wang et al. established an EHL model for materials with a single inhomogeneity, multiple inhomogeneities, functionally graded coatings, and multilayered materials, and the basic behavior of EHL on a heterogeneous interface was studied [7, 8]. Shengguang et al. analyzed the effects of size and position of the inhomogeneities on the EHL lubrication performance and the subsurface stress distribution [9]. Dong and Zhou obtained a numerical solution of heterogeneous materials with multiple inclusions under mixed EHL [10]. Zhao et al. proposed an approach to determine the lubrication performance of the soft EHL of heterogeneous materials [11]. Chen et al. tried to obtain the best lubrication performance of EHL to optimize the related parameters of inhomogeneous materials [12].

In most of the current studies, the contact surfaces of EHL with inhomogeneous particles are assumed to be smooth. However, in reality, the surfaces of two components in contact are naturally rough. In the studies of the life and failure of lubricated contact pairs, the focus has always been on surface roughness $[13,14]$. The rough morphology of a surface can directly affect the contact shape of the thin film, and many scholars have studied the EHL contact on rough surfaces. Tallian observed the disturbance of pressure caused by roughness through numerical simulations and concluded that the roughness may aggravate the surface plastic deformation and failure in an EHL contact [15]. Zhu presented a numerical solution of the effects of the surface roughness on the pressure spike and the film constriction [16]. The surface roughness could not only affect the distribution of film thickness and pressure but also destroy the film in the lubricated contact, which may cause mixed lubrication $[17,18]$. The interaction of asperities and friction force in the movement of a lubricated contact can be affected by the roughness, and this is disadvantageous for the improvement of an EHL environment workings under a heavy load [19].

A rough topography is an important factor that affects the friction force in a lubricated contact, especially an EHL contact with a thin oil film, where the rupture of the oil film can be caused in EHL. The properties of the material properties can be altered by its internal inhomogeneities during the contact process, and the surface displacement and internal stress distribution of the matrix will be changed by the existence of inside particles. The performance of the EHL film thickness, pressure, and shear force between oil and the contact surfaces are related to the surface topography and the material properties. Therefore, considering the influences of both inhomogeneous materials and surface rough topography on EHL, it is meaningful to explore an effective method to reduce the friction of heavy-load lubricated contacts and improve the lubrication environment in actual production.
In the present study, a model is constructed of a half space containing inclusions in contacts with an infinite cylinder. The contact surface is $x_{2}=0$, as shown in Figure 1 . A series of inclusions are buried under the surface of the half space at depth $D$, the side length of the particles is $L$, and the distance between two adjacent particles is $H_{s}$. The upper contact surface is considered to be smooth, while the lower contact surface, $x_{2}=0$, has three different types of rough topography (sinusoidal, saw tooth, and square ripple). Based on the theoretical model, the EHL behaviors of the different types of rough surfaces and inhomogeneous materials are analyzed. Moreover, the effects of external loads, moving speeds, and initial oil viscosities on the performance of the rough EHL are also investigated.

\section{Mathematical Model}

A line contact happened between an infinitely long cylinder $S_{C}$ and a half space $x_{1} o x_{2}$ in the coordinate system of $o x_{1} x_{2}$. The half space contains $n$ arbitrarily shaped inclusion domains $\Omega_{\psi}(\psi=1,2, \ldots, n)$ under the contact surface $x_{2}=0$, as shown in Figure 2, and the material properties of the subdomain $\Omega$ differ from that of the matrix. According to the principle of EIM proposed by Eshelby [2], the inhomogeneous particles can be equivalent to an area that has the same elastic modulus as that of the matrix but subject to eigenstrains e. The basic principle of EIM is shown in Figure 3 .

As the elastic modulus of particle $E_{1}<E_{m}$, the equivalent area will be subjected to a negative eigenstrain. In contrast, the inclusion domain $\Omega_{2}$ has a larger elastic modulus $E_{2}>E_{m}$ and will bear a positive eigenstrain after the equivalence.

\section{Governing Equation for EHL on Rough Surface with Particles}

The whole problem is intercepted by the $x_{1} o x_{2}$ plane which is the main plane, and the analytical model is built and solved. The material properties are given by Lamé constants $\lambda$ and $\mu$ and Poisson's ratio $\nu$. Vector $\mathbf{x}$ denotes the response point on the surface of the half space (lower contact surface), while $\mathbf{x}$ /denotes an excitation point inside the equivalent particle area, as shown in Figure 3. To simplify the expression, $V=1-v$ is used in the equations.

3.1. Surface Displacement Caused by the Particles. According to the elastic field of the Eshelby problem, the displacement of the response point $\left(x_{1}, x_{2}\right)$ caused by particles inside the matrix can be written as

$$
u_{i}(\mathbf{x})=-\int_{-\infty}^{+\infty} C_{j k}^{0} G_{i j, k}\left(\mathbf{x}-\mathbf{x}^{\prime}\right) \mathbf{e d} \mathbf{x}^{\prime},
$$

where $G_{i j}\left(\mathbf{x}-\mathbf{x}^{\prime}\right)$ is Green's function, which can be presented as

$$
G\left(\mathbf{x}-\mathbf{x}^{\prime}\right)=\frac{1}{4 \pi \mu} \frac{\delta_{i j}}{R}-\frac{1}{16 \pi \mu V} \frac{\partial^{2}}{\partial x_{i} \partial x_{j}} R,
$$




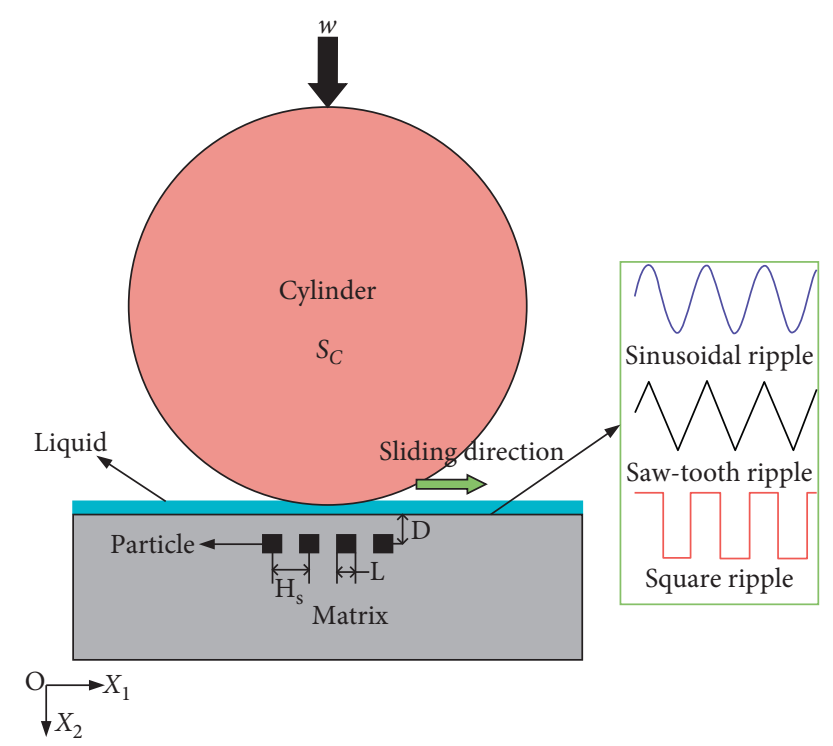

FIgURE 1: EHL in line contact with different rough surfaces.

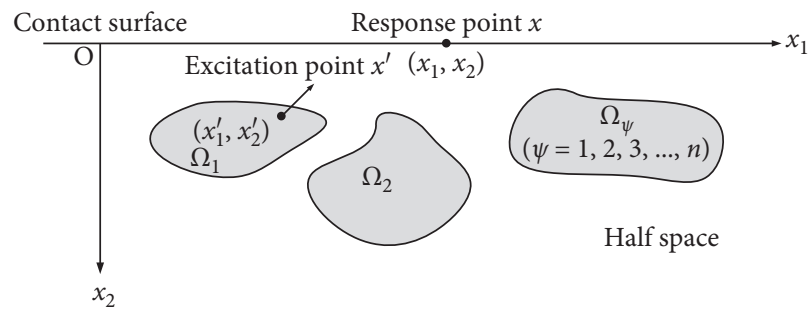

FIGURE 2: Half space with $n$ arbitrary-shaped inclusion domains $\Omega_{\psi}(\psi=1,2, \ldots, n)$.

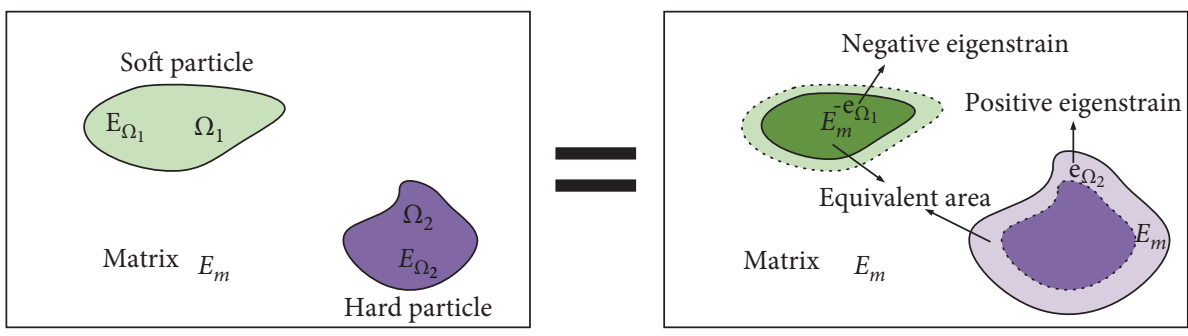

FIgURE 3: Principle of equivalent inclusion.

where $R=\left|\mathbf{x}-\mathbf{x}^{\prime}\right|=\sqrt{\left(x_{1}-x_{1}^{\prime}\right)^{2}+\left(x_{2}-x_{2}^{\prime}\right)^{2}}$ and $\delta_{i j}$ is the Kronecker delta, $\delta_{i j}=0(i \neq j)$ and $\delta_{i j}=1(i=j)$. Equation (2) is substituted into equation (1) to modify the displacement equation as follows:

$$
u_{i}(\mathbf{x})=\frac{1}{8 \pi V}\left[\overline{\bar{\psi}}_{, i}-2 v \Phi_{1}-2 V \Phi_{2}\right] \text { e. }
$$

The simplified vectors in equation (3) are defined as follows:

$$
\begin{aligned}
\overline{\bar{\psi}} & =\left[\psi_{, 11}(\mathbf{x}) ; \psi_{, 22}(\mathbf{x}) ; \psi_{, 12}(\mathbf{x})\right]^{T}, \\
\boldsymbol{\Phi}_{1} & =\left[\Phi_{, 2}(\mathbf{x}), \Phi_{, 2}(\mathbf{x}), 0\right]^{T}, \\
\boldsymbol{\Phi}_{2} & =\left[0,2 \Phi_{, 2}(\mathbf{x}), \Phi_{, 1}(\mathbf{x})\right]^{T},
\end{aligned}
$$

where $\psi(\mathbf{x})=\int_{A} R \mathrm{~d} \mathbf{x}^{\prime}$ and $\Phi(\mathbf{x})=\int_{A}(1 / R) \mathrm{d} \mathbf{x}^{\prime} ;$ the expressions used in the equation denote the derivative with respect to the response point, $\mathbf{x}$, and are defined as $\mathbf{g}_{, i}=\left(\partial \mathbf{g} / \partial x_{i}\right)$.

The surface displacement caused by the particles can be obtained by distributing the response point $\mathbf{x}$ on the contact surface $x_{2}=0$ through equation (3), and it can be written as

$$
\begin{aligned}
u_{s}\left(x_{1}\right) & =\frac{1}{8 \pi V} \mathbf{U}_{s} \mathbf{e}, \\
\mathbf{U}_{s} & =\left(\begin{array}{c}
\psi_{, 112}(\mathbf{x})-2 v \Phi_{, 2}(\mathbf{x}) \\
\psi_{, 222}(\mathbf{x})-2(v+2 V) \Phi_{, 2}(\mathbf{x}) \\
\psi_{, 112}(\mathbf{x})-2 V \Phi_{, 1}(\mathbf{x})
\end{array}\right) .
\end{aligned}
$$


3.2. The Reynolds Equation. Considering that the fluid between the contact surfaces has the properties of a Newtonian fluid, the temperature changes in the flow field are ignored. The one-dimensional generalized Reynolds equation for describing flow problems in narrow gaps can be written as

$$
\frac{\mathrm{d}}{\mathrm{d} x_{1}}\left(\varepsilon \frac{\mathrm{d} p}{\mathrm{~d} x_{1}}\right)-12 u_{1} \frac{\mathrm{d}(\rho h)}{\mathrm{d} x_{1}}=0,
$$

where $\varepsilon=\left(\rho h^{3} / \eta\right), \eta$ is the viscosity, $\rho$ is the density of the fluid, and $u_{1}$ is the speed of the upper contact sliding on the surface of the half space along the $x_{1}$-axis.

The nondimensional form of equation (6) is given by

$$
\frac{\mathrm{d}}{\mathrm{d} X_{1}}\left(\varepsilon \frac{\mathrm{d} P}{\mathrm{~d} X_{1}}\right)-\frac{\mathrm{d}\left(\rho^{*} H\right)}{\mathrm{d} X_{1}}=0
$$

The nondimensional parameters for equation (6) are $W=\left(w / E^{\prime} R_{s_{c}}\right), \quad H=\left(h R_{s_{c}} / b^{2}\right), \quad P=\left(p / p_{0}\right), \quad U_{1}=\left(\eta_{0} u_{1} /\right.$ $\left.E^{\prime} R_{s_{c}}\right)$, and $X_{1}=\left(x_{1} / b\right)$.

3.3. Film Thickness Equation. The film thickness equation not only includes the deformed part of the contact body and the contact geometry but also considers the surface deformation caused by the particles and the roughness of the contact surface. Combining the factors mentioned above, the film thickness at any response point on the contact surface can be written as

$$
\begin{aligned}
h\left(x_{1}\right)= & h_{0}+\frac{x_{1}^{2}}{2 R_{s_{c}}}-\frac{2}{\pi E^{\prime}} \int_{x_{1}-\text { in }}^{x_{1}-\text { out }} p(s) \ln \left(x_{1}-s\right) \mathrm{d} s \\
& +u_{s}\left(x_{1}\right)+r_{s}\left(x_{1}\right),
\end{aligned}
$$

where $h_{0}$ is the central film thickness, $R_{S_{c}}$ is the equivalent radius of curvature in the $x_{1}$ direction, $E^{\prime}$ is the equivalent elastic modulus of the contact area, $u_{s}\left(x_{1}\right)$ is the surface displacement caused by the particles inside the matrix, and $r_{s}\left(x_{1}\right)$ is the surface roughness, and three types of roughness are adopted sinusoidal, saw tooth, and square. It can be expressed as follows.

For the sinusoidal ripple roughness,

$$
r_{s}\left(x_{1}\right)=A_{r}\left(\sin \left(\frac{2 \pi}{\lambda_{r}} x_{1}\right)+1\right) \text {. }
$$

For the saw-tooth ripple roughness,

$$
r_{s}\left(x_{1}\right)=\frac{2 A_{r}}{\pi}\left(\arcsin \left(\sin \left(\frac{2 \pi}{\lambda_{r}} x_{1}\right)\right)+\frac{1}{2 \pi}\right) \text {. }
$$

For the square ripple roughness,

$$
r_{s}\left(x_{1}\right)=A_{r} \operatorname{sgn}\left(\sin \left(\frac{2 \pi}{\lambda_{r}} x_{1}\right)\right),
$$

where $\operatorname{sgn}(\theta)$ is a symbolic function expressed as

$$
\operatorname{sgn}(\theta)= \begin{cases}1, & \text { as } \theta>0, \\ 0, & \text { as } \theta \leq 0,\end{cases}
$$

where $A_{r}$ is the roughness amplitude and $\lambda_{r}$ is the wavelength of the roughness.
Equation (8) in the nondimensional form is given by

$$
\begin{aligned}
H\left(X_{1}\right)= & H_{0}+\frac{X_{1}^{2}}{2}-\frac{1}{\pi} \int_{X_{1-\text { in }}}^{X_{1-\text { out }}} \ln \left|X_{1}-X_{1}^{\prime}\right| P\left(X_{1}^{\prime}\right) \mathrm{d} X_{1}^{\prime} \\
& +U_{s}\left(X_{1}\right)+R_{s}\left(X_{1}\right) .
\end{aligned}
$$

3.4. Nondimensional Density Pressure Relationship. Ignoring the changes in temperature, the most general relationship between density and pressure is

$$
\rho=\rho_{0}\left(1+\frac{0.6 \times 10^{-9} P}{1+1.7 \times 10^{-9} P}\right) .
$$

Equation (14) in a nondimensional form is given by

$$
\rho^{*}=1+\frac{0.6 \times 10^{-9} P}{1+1.7 \times 10^{-9} P},
$$

where $\rho_{0}$ is the density of the liquid at atmospheric pressure.

3.5. Nondimensional Viscosity-Pressure Relationship. The viscosity-pressure relationship can use the equation given by Roelands [20]:

$$
\eta=\eta_{0} \exp \left\{\left[\ln \left(\eta_{0}\right)+9.67\right]\left[-1+\left(1+5.1 \times 10^{-9} p\right)^{z}\right]\right\} .
$$

Its nondimensional form can be written as

$$
\eta^{*}=\exp \left\{\left[\ln \left(\eta_{0}\right)+9.67\right]\left[-1+\left(1+5.1 \times 10^{-9} p\right)^{z}\right]\right\} \text {, }
$$

where $\eta_{0}$ is the viscosity of the liquid at atmospheric pressure and $z$ is the experiment constant, which can be determined by the coefficient of Barus viscosity-pressure relationship $\alpha$ [21]:

$$
z=\frac{\alpha}{5.1 \times 10^{-9}\left(\ln \eta_{0}+9.67\right)} \text {. }
$$

3.6. Force Balance Equation. The sum of the pressures in the liquid in the flow field is balanced with the external forces $w$ :

$$
w=\int_{x_{1}-\text { in }}^{x_{1}-\text { out }} p\left(x_{1}\right) \mathrm{d} x_{1} .
$$

Equation (18) in the nondimensional form is given as

$$
\int_{X_{1-\text { in }}}^{X_{1-\text { out }}} P\left(X_{1}\right) \mathrm{d} X_{1}=\frac{\pi}{2} .
$$

The nondimensional corresponding boundary conditions are as follows:

Inlet boundary condition at $P=0$

Outlet boundary condition at $X_{1}=X_{1 \text {-out }}$ and $P=\mathrm{d} P /$ $\mathrm{d} X_{1}=0$

\section{Results}

The parameters used in the calculation are listed in Table 1. According to Eshelby's EIM, the material of inhomogeneous 
TABLE 1: Input parameters.

\begin{tabular}{lc}
\hline Equivalent elastic modulus of the & $2.21 \times 10^{11} \mathrm{~Pa}$ \\
composites $E^{\prime}$ & $2.0 \times 10^{-8} \mathrm{~Pa}^{-1}$ \\
Barus pressure-viscosity coefficient, $\alpha$ & $100 \mathrm{~N}$ \\
Applied load, $w$ & $0.3 \mathrm{GPa}$ \\
Maximum Hertzian pressure, $P_{h}$ & $0.05 \mathrm{~m}$ \\
Equivalent radius, $R_{s_{c}}$ & $0.05 \mathrm{~Pa} \cdot \mathrm{s}$ \\
Inlet viscosity of fluid, $\eta_{0}$ & $-2.5 \leq X_{1} \leq 1.5$ \\
The calculation domain, $X_{1}$ & 0.03 \\
Dimensionless step length, $\Delta X_{1}$ & $0.2 \mathrm{~mm}$ \\
Particle side length, $L$ & $0.45 \mathrm{~mm}$ \\
Adjacent particles spacing, $H_{s}$ & $0.2 \mathrm{~mm}$ \\
Particle burial depth, $D$ & $0.12 \mathrm{~mm}$ \\
Wavelength of waviness, $\lambda_{r}$ & $0.1 \mathrm{~mm}$ \\
Amplitude of waviness, $A_{r}$ & 0 \\
Velocity of the half space in $X_{1}$ direction, $U_{m}$ & $1.0 \mathrm{~m} / \mathrm{s}$ \\
Velocity of the cylindrical contact in $X_{1}$ & $1.65 \times 10^{11} \mathrm{~Pa}$ \\
direction, $U_{x_{1}}$ & $2.5 \times 10^{11} \mathrm{~Pa}$ \\
Elastic modulus of half space, $E_{1}$ & 0.3 \\
Elastic modulus of cylindrical contact, $E_{2}$ & 0.34 \\
Poisson's ratio of half-space, $\nu_{1}$ & 0.25 \\
Poisson's ratio of cylindrical contact, $v_{2}$ & $0.003 \times[1,1,2] \mathrm{mm}$ \\
Poisson's ratio of particles, $v$ & \\
Eigenstrains, e &
\end{tabular}

inclusions can be the same as the material of the matrix and the equivalent area should have eigenstrains. The eigenstrains are positive if the inhomogeneous inclusions are harder than the matrix, otherwise they are negative. In this study, the eigenstrains are expressed in one-dimensional form $\mathbf{e}=\left[e_{11}, e_{22}, 2 e_{12}\right]$. The effects of eigenstrains of the particles and the rough topography of the contact surface on the behaviors of the EHL are explored and the results are as follows.

4.1. Effects of the Sinusoidal Roughness on the EHL with Particles. Four particles are buried under the sinusoidal surface, and the effects of the hard particles with eigenstrains e and the soft particles with eigenstrains -e on the EHL film thickness and pressure are shown in Figures 4(a) and 4(b)), respectively. From Figure 4(a), the film thickness of the soft particles is smaller than that of the hard particles at the inlet area, in contact surfaces with the same roughness. Compared with the situation of a smooth contact surface without particles, the film thickness at the position of the soft particles located is greater. Film thickness at the area between the soft particles is smaller than that with no inclusions, but the reduction is not significant. It is worth noting that the equivalent film thickness of the soft particles in the contact zone is higher than that in the case no inclusions, whereas for hard particles this is the opposite. The minimum film thickness of the sinusoidal surface with particles (soft or hard) begins to decrease at the outlet and it is smaller than that of the smooth surface without particles. The minimum film thickness, $H_{\text {sin-h1-min }}$ of the hard particles is nearly equal to that of the soft particles, $H_{\text {sin-s1-min }}=0.2$.

Figure 4(b) compares the pressure of the soft and hard particles under the sinusoidal rough contact surface. The pressure at the location of the hard particles is greater than that of a smooth surface with no particles, whereas the situation with the soft particles is the opposite. At the area between the particles the pressure of the soft particles is much greater than that of the hard particles. The maximum pressure appears at the contact center for the hard particles, $P_{\text {sin-h1-max }}=1.28$. For the soft particles, the pressure at the contact center is $P_{\mathrm{sin}-\mathrm{s} 1}=0.75$, and the maximum pressure of the soft particles appears at the outlet area $P_{\text {sin-s1-max }}=1$. It is obvious that at the outlet area the pressure of the hard particles is much smaller than that in the case with the no inclusions, whereas the presence of soft particles increases the film pressure.

The film thickness and the pressure of the sinusoidal rough contact surface when the particle area has the eigenstrains, 2e and $-2 \mathbf{e}$, are shown in Figures 5(a) and 5(b)), respectively. Comparing Figures 4(a) and 5(a), it can be found that as the particles become harder or softer, the film thickness at the position where the particles are located fluctuates much more. Different from the situation with eigenstrains $\mathbf{e}$ and $-\mathbf{e}$, the outlet film thickness of particles with eigenstrains $2 \mathbf{e}$ is thinner, whereas the film thickness of $-2 \mathbf{e}$ is increased.

Comparing Figures 4(b) and 5(b), it can be observed that the maximum pressure of the hard particles with eigenstrain 2e, $P_{\text {sin-h2-max }}=1.6$, is much greater than that of $P_{\text {sin-h1-max }}$. For the soft particles with eigenstrain $-2 \mathbf{e}$, the center pressure, $P_{\text {sin-s } 2}=0.65$, is much lower than $P_{\text {sin-s } 1}$, the maximum pressure also appears near the outlet $P_{\text {sin-s2-max }}=1.15$, and it is slightly larger than $P_{\text {sin-s1-max }}$.

\subsection{Effects of the Saw-Tooth Roughness on the EHL with} Particles. Figure 6(a) shows the film thickness of the sawtooth contact surface, where the eigenstrains of particles are $\mathbf{e}$ and -e. Compared with the sinusoidal surface, the local film shape becomes sharper, and the fluctuation of the oil film caused by the particles is relatively small. The equivalent film thickness in the inlet and outlet zones is almost the same. The equivalent film thickness of the soft particles in the contact area is increased compared with that of the smooth surface without particles, whereas the situation is the opposite for the hard particles. The minimum film thickness of the soft particles, $H_{\text {saw-s1-min }}$ in the outlet area is approximately equal to that of the hard particles, $H_{\text {saw-h1-min }}=0.18$.

Figure 6(b) shows the pressure of the particles with eigenstrains $\mathbf{e}$ and $-\mathbf{e}$ under the saw-tooth surface. For the hard particles, the maximum film pressure, $P_{\text {saw-h1-max }}=1.3$, is increased compared to that of the sinusoidal contact surface. The center film pressure at the location of the soft particle decreases to $P_{\text {saw-s } 1}=0.7$, and the maximum film pressure also appears at the outlet area $P_{\text {saw-s1-max }}=1.1$. $P_{\text {saw- }}$ s1-max, is increased compared to $P_{\text {sin-s1-min }}$ of the sinusoidal rough surface with the same particles.

Figure 7 (a) shows the nondimensional film thickness of particles with eigenstrains $2 \mathbf{e}$ and $-2 \mathbf{e}$ under the saw-tooth surface. Comparing Figure 6(a) with Figure 7(a), it can be found that the equivalent film thickness of soft particles with eigenstrain $-2 \mathbf{e}$ is obviously increased and lager than that of 


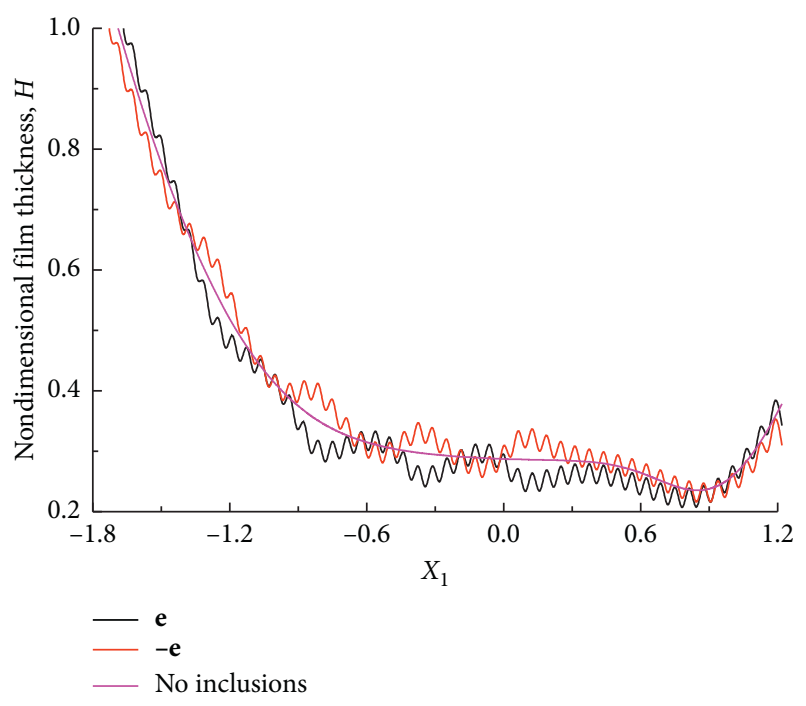

(a)

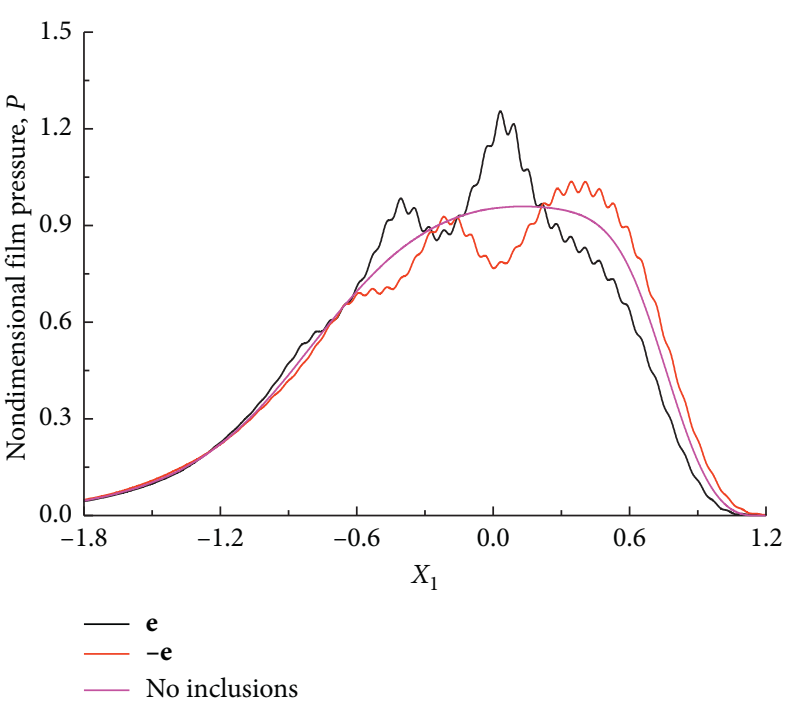

(b)

FIGURE 4: Nondimensional film thickness and pressure of the sinusoidal rough surface with the particle eigenstrains, e and -e.

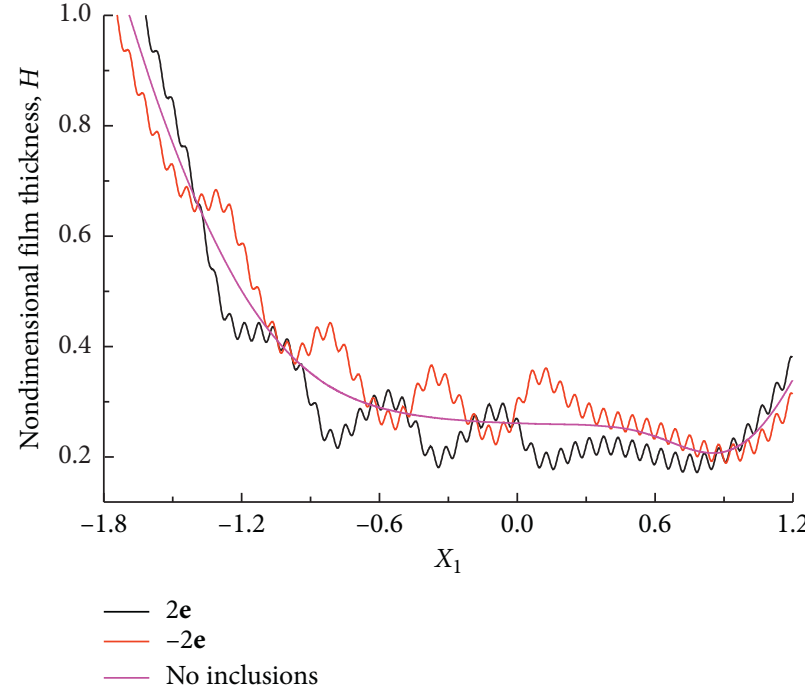

(a)

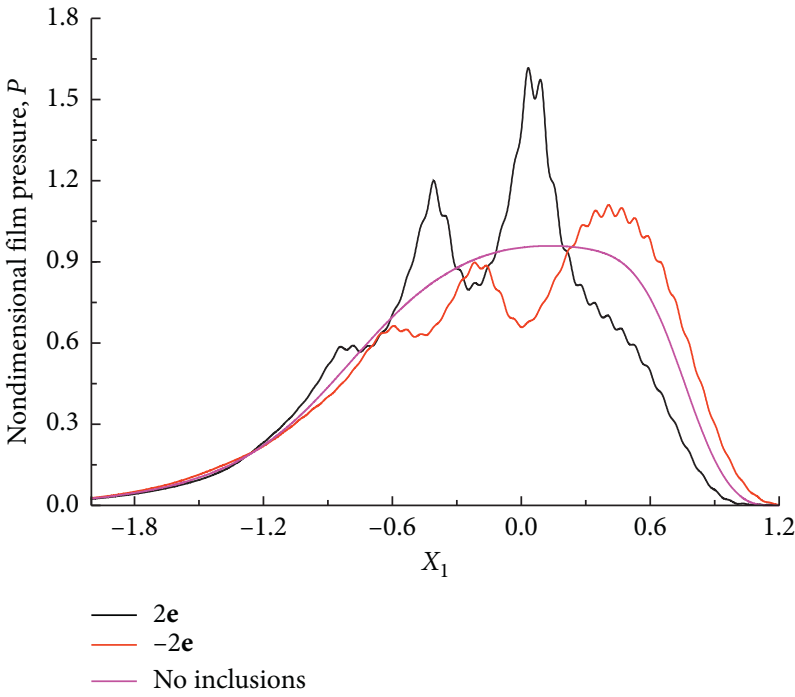

(b)

Figure 5: Nondimensional film thickness and pressure of the sinusoidal rough surface with particle eigenstrains, $2 \mathbf{e}$ and $-2 \mathbf{e}$

the smooth surface without particles, whereas the center film thickness gets smaller as the hard particle eigenstrain increases. Different from the sinusoidal contact surface, the film thickness at the location between particles is almost the same.

Figure 7(b) shows the nondimensional film pressure of particles with eigenstrains $2 \mathbf{e}$ and $-2 \mathbf{e}$ under the saw-tooth contact surface. Comparing Figure 6(b) with Figure 7(b), it can be found that the maximum pressure of the hard particles with eigenstrain $2 \mathbf{e}, P_{\text {saw-h2-max }}=1.7$, is greater than that of $P_{\text {saw-h1-max }}=1.3$. From Figures 5(b) and 7(b), it can be observed that the effect of the same particles on pressure has no differences, although the surface rough topography is different. As in other cases, the film pressure of hard particles begins to decrease near the outlet area, whereas the center film pressure of the soft particles, $P_{\text {saw-s2 }}$, decreases to 0.6 , which is lower than that of $P_{\text {saw-s1 }}$ in Figure 6(b). The maximum film pressure of the soft particles, $P_{\text {saw-s2-max }}=1.15$, is almost equal to $P_{\text {sin-s2-max }}$ but slightly larger than $P_{\text {saw-s1-max }}$.

\subsection{Effects of the Square Roughness on the EHL with Particles.} Figure 8(a) shows the film thickness of particles with eigenstrains $\mathbf{e}$ and $-\mathbf{e}$ under the square contact surface. In the same way, as in the sinusoidal and saw-tooth rough surfaces, shown in Figures 4(a) and 6(a), the film thickness at the position of the hard particles is decreased compared with the situation in the smooth contact surface with no particles, whereas it is the 


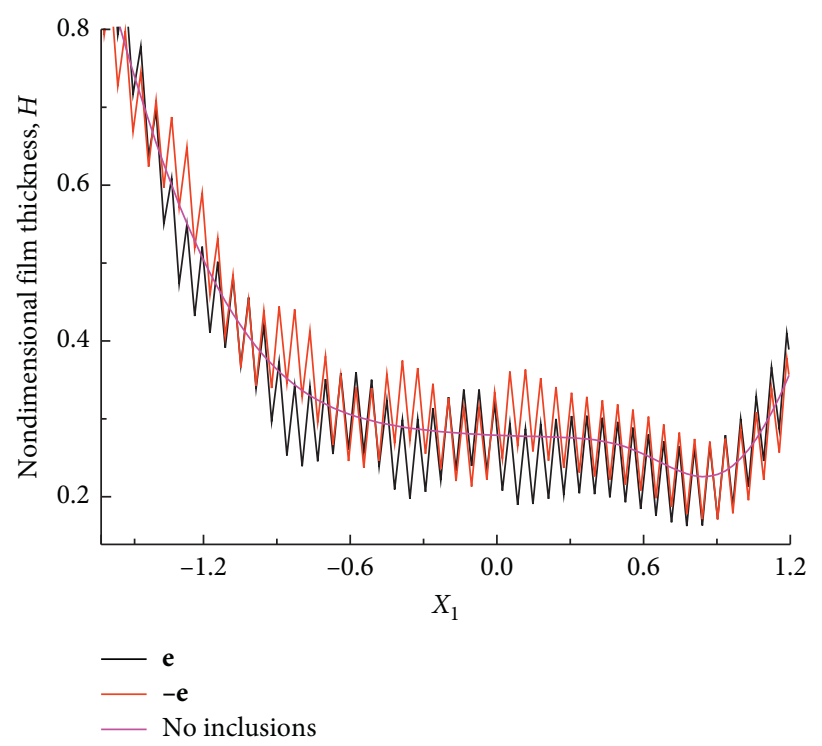

(a)

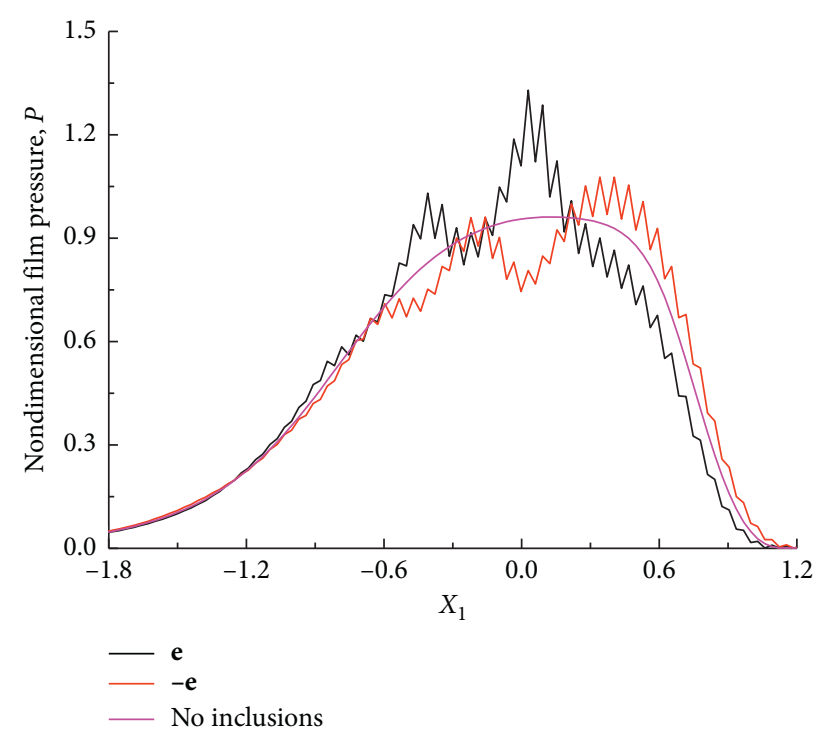

(b)

FIgURE 6: Nondimensional film thickness and pressure of the saw-tooth rough surface with particle eigenstrains, e and -e.

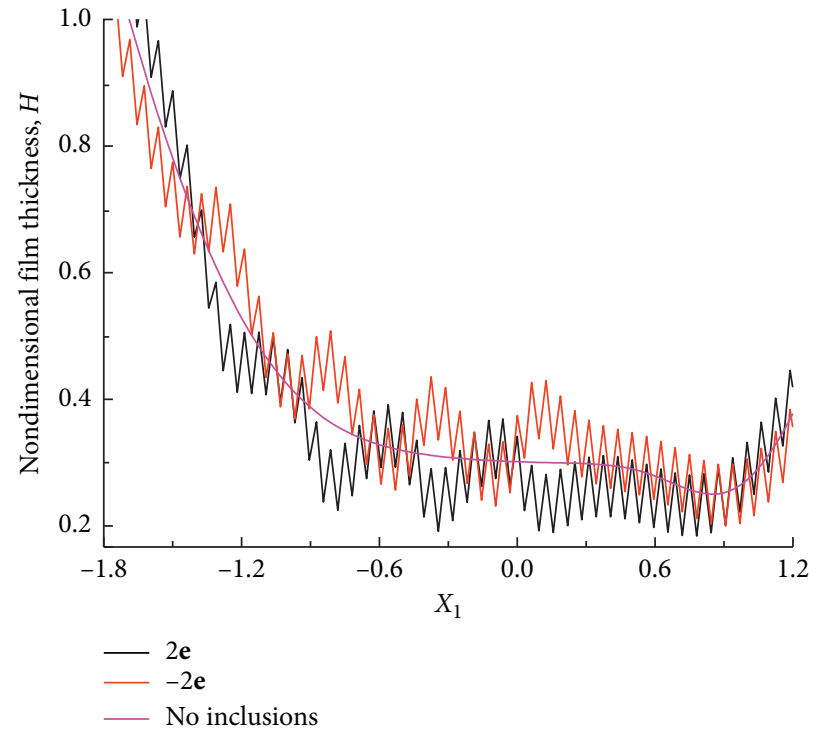

(a)

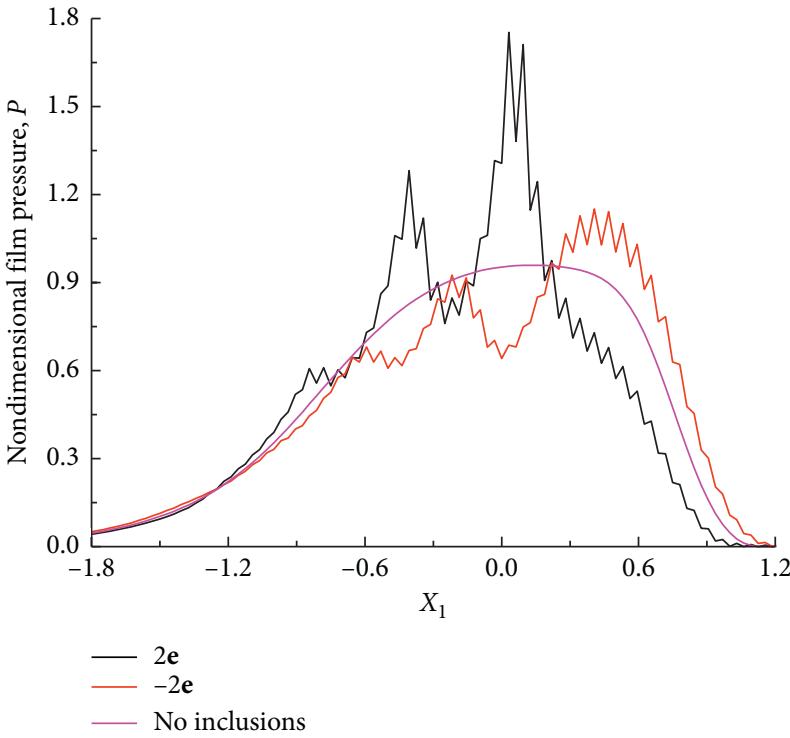

(b)

Figure 7: Nondimensional film thickness of the saw-tooth rough surface with particle eigenstrains, $2 \mathbf{e}$ and $-2 \mathbf{e}$.

opposite for the soft particles. The minimum film thickness appears at the outlet area for both of hard and soft particles, and their values are $H_{\text {sq-h1-min }}=H_{\text {sq-s1-min }}=0.21$.

Figure $8(\mathrm{~b})$ shows the film pressure of particles with eigenstrains $\mathbf{e}$ and $-\mathbf{e}$ under the square contact surface. For the hard particles, the maximum film pressure is $P_{\text {sq-h1- }}$ $\max =1.35$, and the maximum pressure of the square surface is increased compared to the sinusoidal and saw-tooth rough surfaces in Figures 4(b) and 6(b). For the soft particles, the maximum film pressure is $P_{\text {sq-s1-max }}=1.1$, and the film center pressure $P_{\text {sq-s } 1}=0.7$ is almost the same as that in the first two rough topography.
The nondimensional film thickness and pressure of the particles with eigenstrains $2 \mathbf{e}$ and $-2 \mathbf{e}$ under the square rough surface is as shown in Figures 9(a) and 9(b). Compared with Figure $8(\mathrm{a})$, the center film thickness, $H_{\mathrm{sq}-\mathrm{h} 2}$, is decreased for the hard particles. However, the film thickness at the positions where the soft particles are located is increased, and the minimum film thickness of soft particles is greater than that of the hard particles.

The maximum pressure of the hard particles appears in the contact center, $P_{\text {sq-h2-max }}=1.8$, as shown in Figure $9(\mathrm{~b})$, and is increased compared with that in the sinusoidal and saw-tooth rough surfaces. For the soft particles, the 


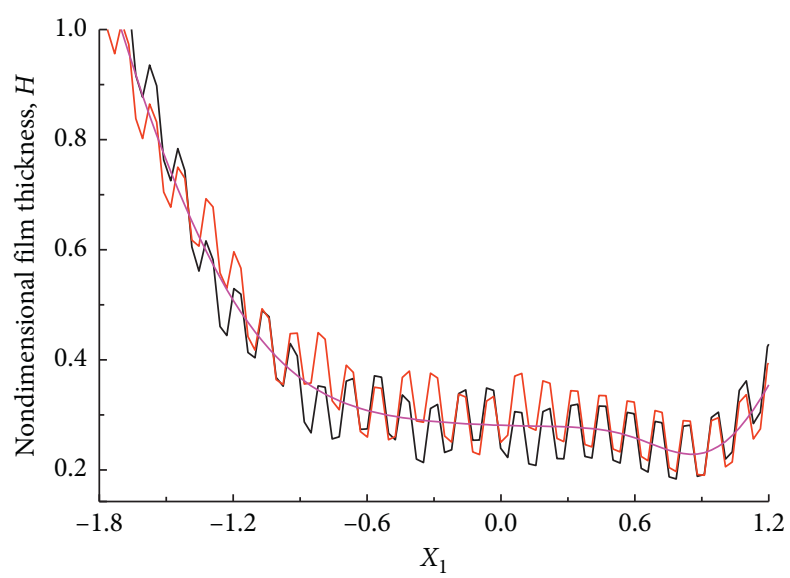

$\mathbf{e}$
$-\mathbf{- e}$
- No inclusions

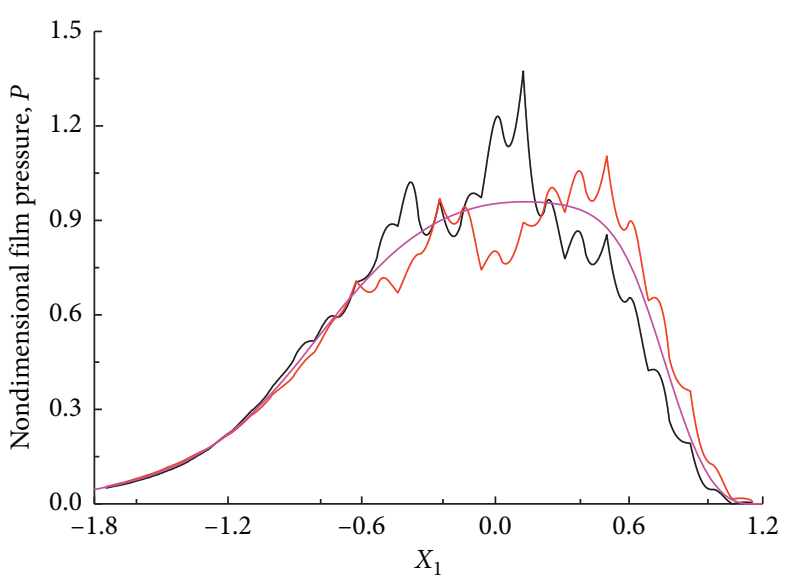

- $\mathrm{e}$

(a)

(b)

Figure 8: Nondimensional film thickness and pressure of the square roughness with eigenstrains, e and $-\mathbf{e}$.

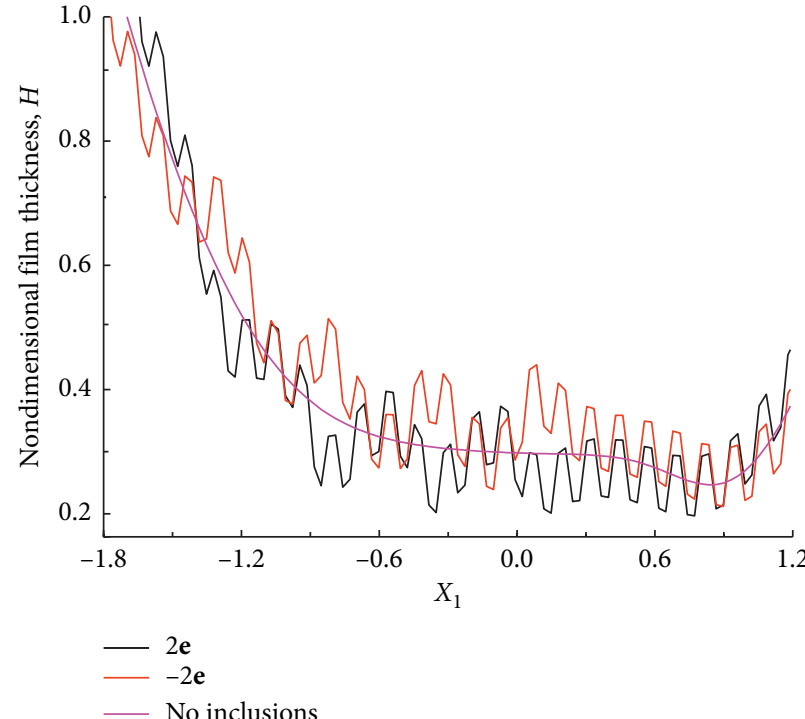

(a)

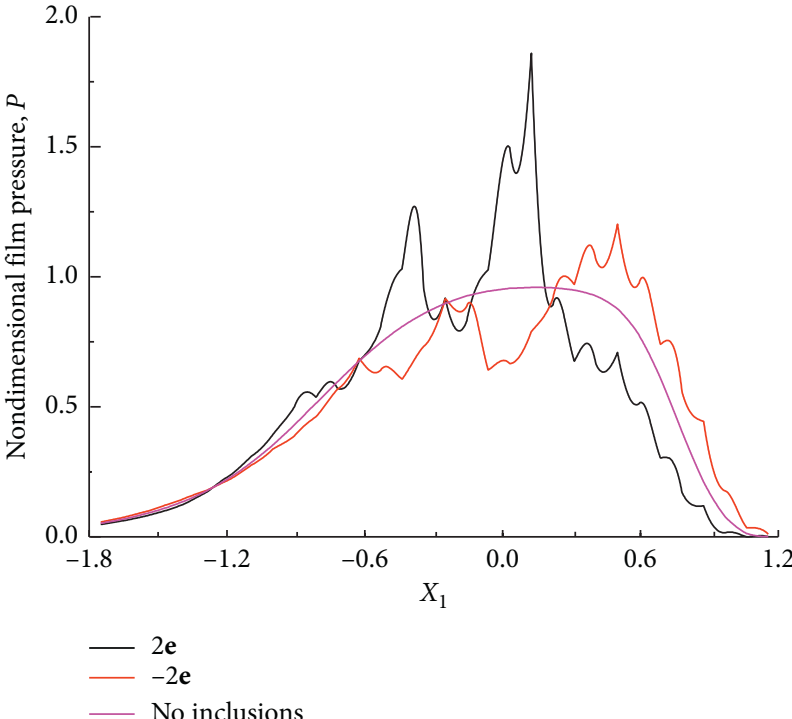

(b)

FIGURE 9: Nondimensional film thickness and pressure of the square roughness with eigenstrains, $2 \mathbf{e}$ and $-2 \mathbf{e}$.

maximum film pressure, $P_{\text {sq-s2-max }}=1.2$, appears at the outlet area, but the pressure in the contact center is decreased to 0.65 compared to the situation with no particles, and it is a little higher than that of the sinusoidal and sawtooth rough surfaces, as shown in Figures 5(b) and 7(b).

4.4. Effects of Roughness on the Traction Force of the EHL. Figure 10 shows the effects of the rough surface topography and roughness on the traction force of the inclusion EHL. The sinusoidal rough surface has the rough wavelength $\lambda_{r}=50 \mu \mathrm{m}$, and the inclusion-EHL traction force with different rough amplitudes is shown in Figure 10(a). It illustrates that the traction force increases with the increase in the roughness amplitude Ra. Figure 10(b) compares the effects of the sinusoidal, saw-tooth, and square rough surfaces on the EHL with different particles, and the traction force is $T_{f}$ (square) $<T_{f}$ ( sinusoidal) $<T_{f}$ (saw - tooth) with particles of the same characteristics. Compared with the case of no particles, the traction force of the EHL with hard particles is increased, whereas it is reduced with the presence of soft particles.

4.5. Effects of Particle Parameters on the Traction Coefficient of the EHL. Figure 11 illustrates the effects of the particle burial depth on the traction coefficient of the inclusion-EHL on surfaces with different rough topographies, with particle side 


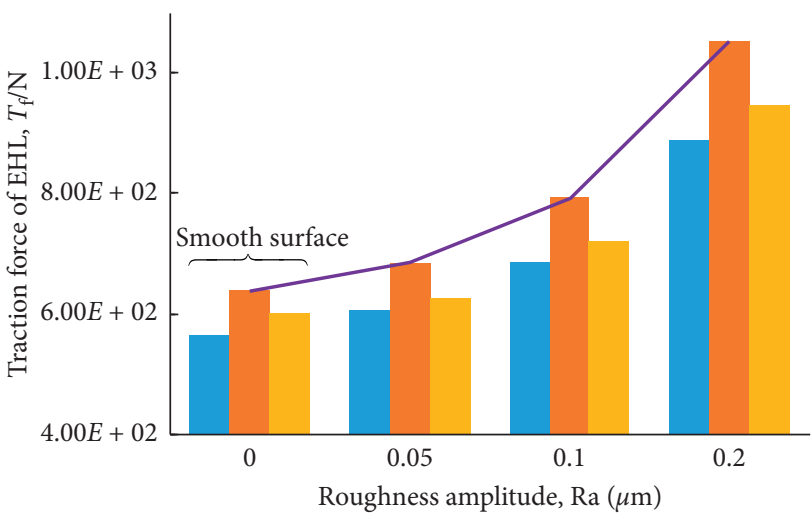

Soft particles

Hard particles

No particles

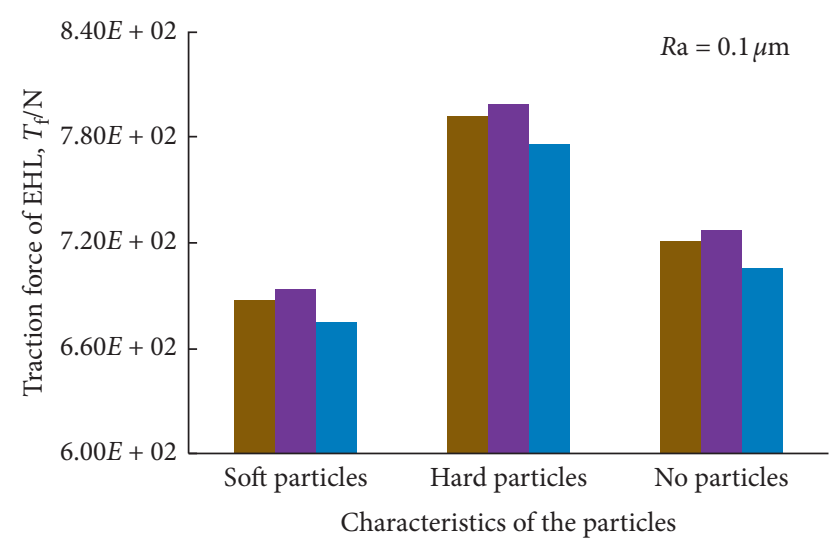

Sinusoidal ripple

Saw-tooth ripple

Square ripple

FIGURE 10: Effects of roughness on the traction force of the EHL: (a) sinusoidal ripple surface with different amplitudes and particles and (b) sinusoidal, saw-tooth, and square ripple surfaces with different particles at the amplitude $\mathrm{Ra}=0.1 \mu \mathrm{m}$.

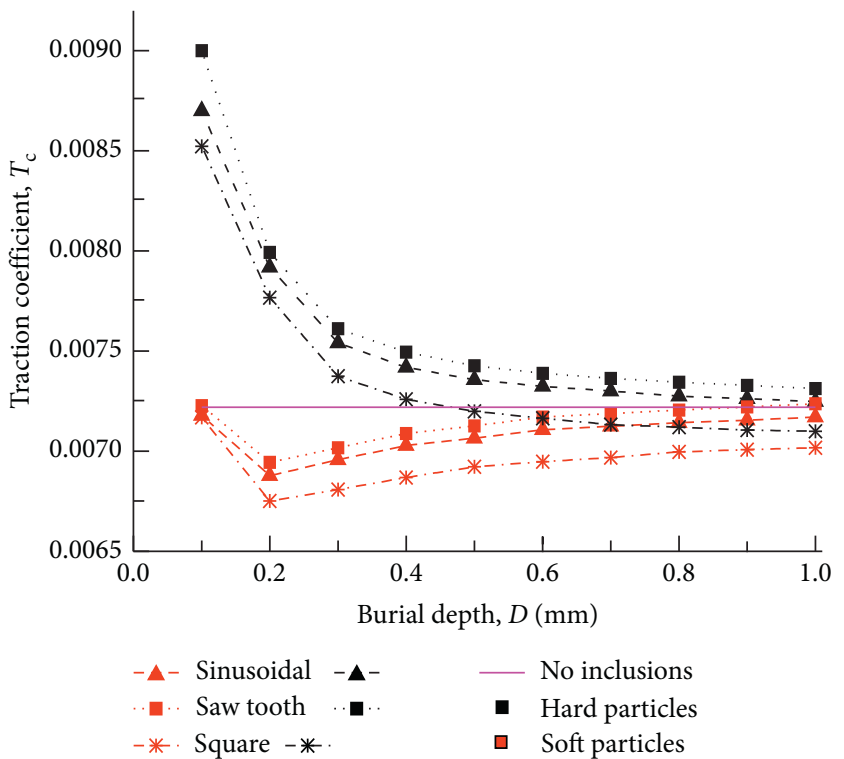

Figure 11: Effects of particle burial depth on the traction coefficient.

length $L=0.2 \mathrm{~mm}$, and particle spacing $H_{s}=0.45 \mathrm{~mm}$. The roughness amplitude is $\mathrm{Ra}=0.1 \mu \mathrm{m}$ and rough wavelength $\lambda_{r}=50 \mu \mathrm{m}$. For the same type of particles, the traction coefficient is $T_{c}$ (Square) $<T_{c}$ (sinusoidal) $<T_{c}$ (Sawtooth). Under the action of the film pressure, the contact surface with soft particles will form a dimple-like structure, as shown in Figures 4, 6, and 8(a), and this type of structure can store oil and improve the EHL environment. Therefore, the traction coefficient $T_{c}$ of the soft particles is reduced compared with the situation of no particles. On the contrary, the existence of the hard particles will form a convex-like structure on the surface, which will reduce the local film thickness and hinder the oil film during the movement. When the particle burial depth $D$ is small, the film local shear force will increase dramatically due to the large deformation of the surface affected by the particles. With the increase in the burial depth, the fluctuation of the surface displacement caused by the particles begins to slow down. For the soft particles, the traction coefficient decreases first and then increases, and it obtains the minimum value at $D=0.2 \mathrm{~mm}$. When the soft particles are buried too deep, the friction coefficient is close to that in the situation of no inclusions. For the hard particles, its traction coefficient decreases with the increase in burial depth, and finally it also approaches to the situation without particles.

Figure 12 shows the effects of particle spacing on the traction coefficient of the inclusion EHL contact, with particle burial depth $D=0.2 \mathrm{~mm}$ and side length $L=0.2 \mathrm{~mm}$. For the hard particles, when the particle spacing $H_{s}<1.0 \mathrm{~mm}$, the traction coefficient, $T_{c}$, decreases with the increase in $H_{s}$, and $T_{c}$ is greater than that in the case of no inclusions when $H_{s}<0.9 \mathrm{~mm}$. When $H_{s}>1.0 \mathrm{~mm}, T_{c}$ begins to increase slowly, and finally its value is almost equal to that of the situation with no inclusions. The change trend in the traction coefficient of soft particles is completely opposite to that of hard particles.

The effects of particle side length $L$ on the traction coefficient $T_{c}$ of inclusion EHL is shown in Figure 13 with particle burial depth $D=0.2 \mathrm{~mm}$ and spacing $H_{s}=0.45 \mathrm{~mm}$. It can be observed from the figure that the fluctuation of the particle side length has little effect on the traction coefficient of EHL when the contact surface has the same rough topography.

4.6. Effect of Applied Load on the Minimum Film Thickness of Different Roughness and Particles. Figure 14 shows the effects of the applied load on the minimum film thickness of particles with eigenstrains $\mathbf{e}$ and $-\mathbf{e}$ under the sinusoidal, saw-tooth, and square contact surface, respectively. When the applied load is low, the minimum film thickness is nearly 


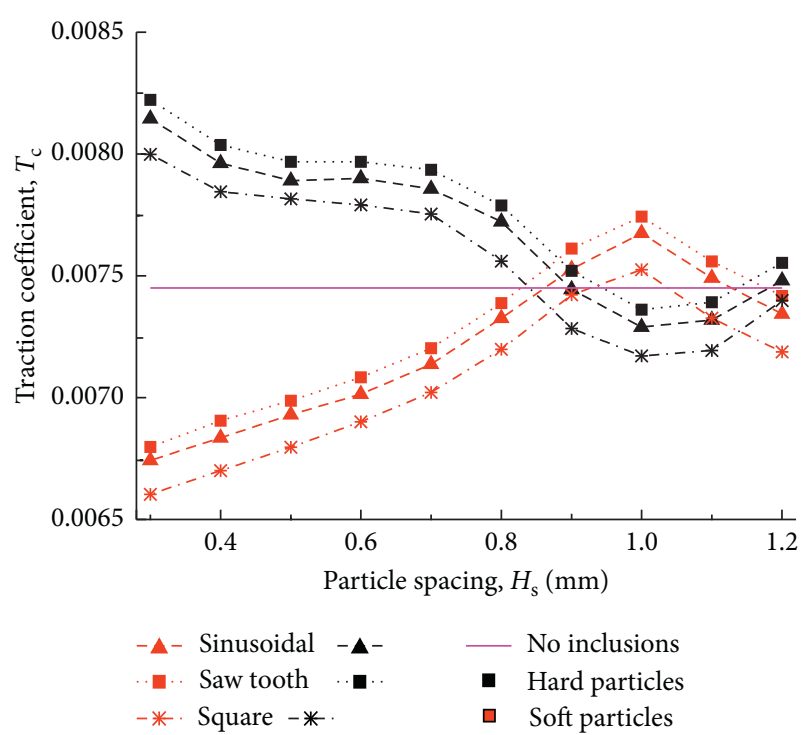

FIGURE 12: Effects of particle spacing on the traction coefficient.

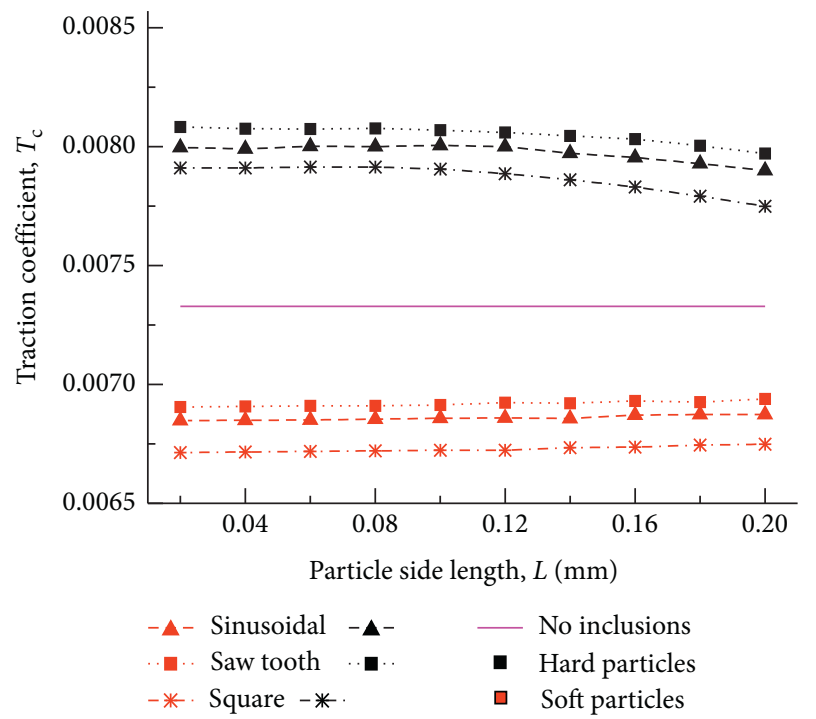

FIgURE 13: Effects of particle side length on the traction coefficient.

equal. As the pressure increases, the minimum oil film thickness gradually decreases. Under the same applied load, the minimum film thickness of the square contact surface is much larger than that of the sinusoidal and saw-tooth surface, and the minimum film thickness of the soft particles is larger than that of the hard particles.

\subsection{Effect of Sliding Speed on the Minimum Film Thickness of} Different Roughness and Particles. Figure 15 shows the effects of the sliding speed on the minimum film thickness. When the speed is small, the $H_{\min }$ of the square contact surface is much larger. As the speed increases, the growth rates of $H_{\min }$ of the soft particles under the sinusoidal and saw-tooth contact surfaces are increased. For the hard particles, the minimum film thickness decreases as the speed increases.

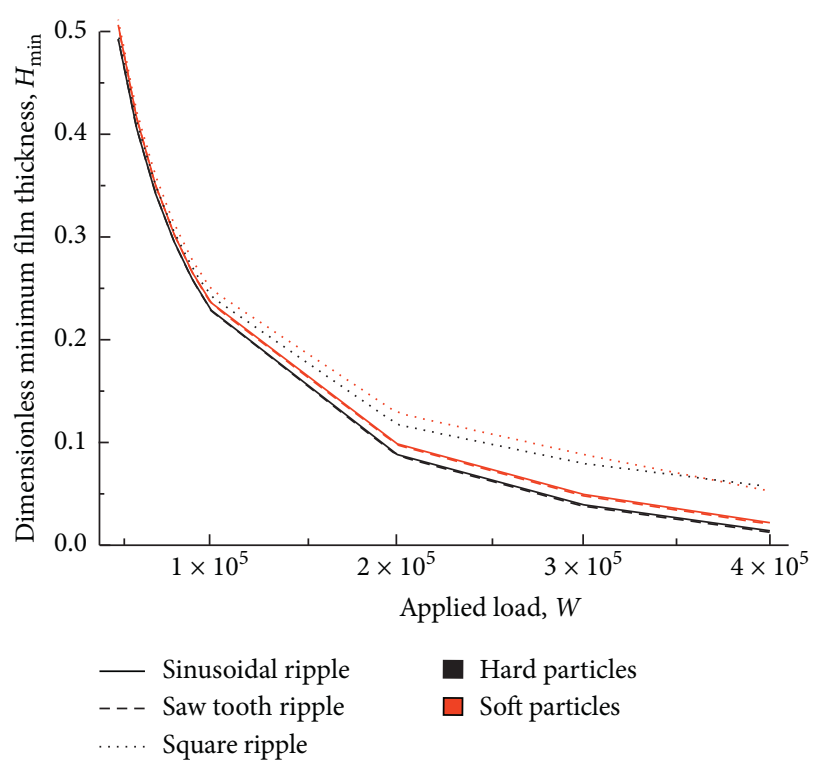

FIGURE 14: Nondimensional minimum film thickness with different applied loads.

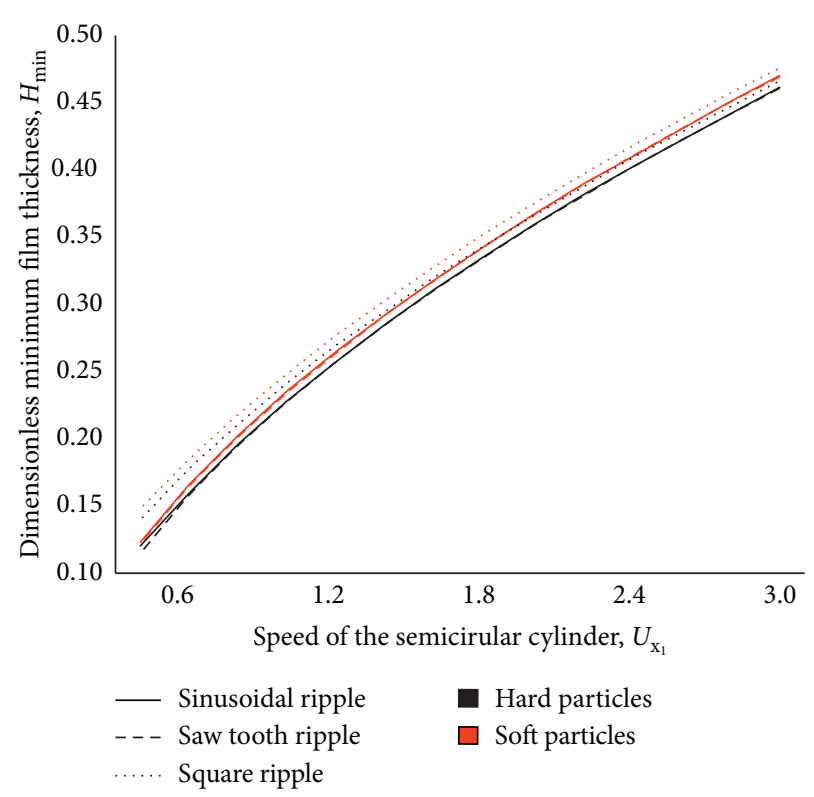

Figure 15: The nondimensional minimum film thickness with different speeds of the semicircular.

4.8. Effect of Initial Viscosity on the Minimum Film Thickness of Different Roughness and Particles. Figure 16 shows the effects of the original viscosity on the minimum film thickness. As the original viscosity increases, the minimum film thickness of the square contact surface with soft particles is the largest. Although the minimum film thickness of the soft particles under the sinusoidal and saw-tooth contact surfaces is increased, they are still smaller than that of the hard particles under the square surface. The minimum film thickness of the hard particles under the sinusoidal and sawtooth surfaces has the smallest values with the change in the original viscosity. 


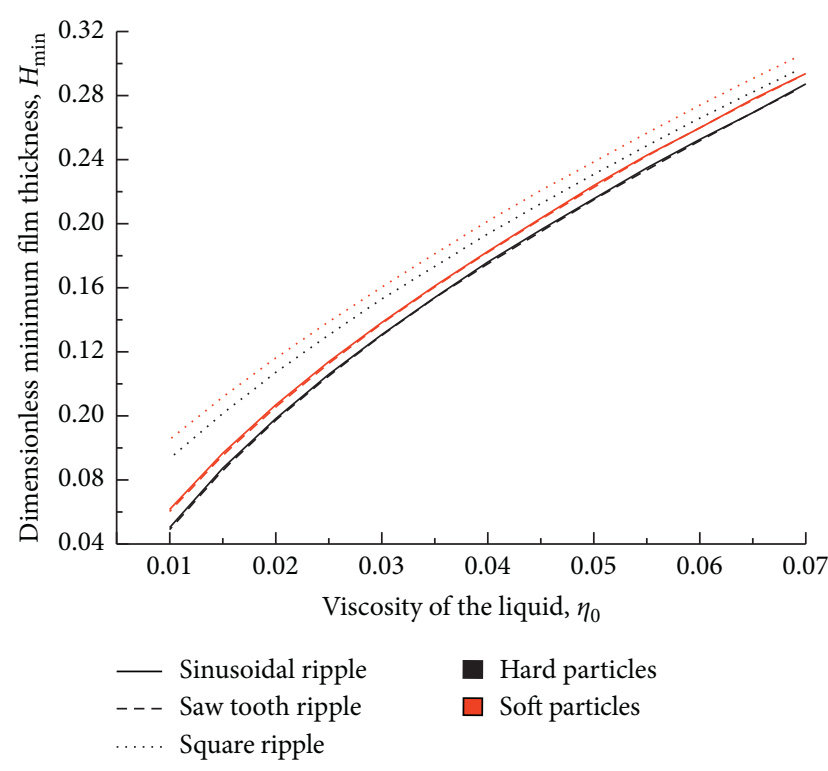

FIgURE 16: Nondimensional minimum film thickness with different initial viscosities.

\section{Conclusions}

In this study, a model of EHL with a series of particles under a rough contact surface is built, where the particles are equivalent to the same material of the matrix with eigenstrains. The effects of different eigenstrains of the particles under sinusoidal, saw-tooth, and square surfaces are analyzed. The following conclusions can be drawn from the study:

(1) The minimum film thickness of EHL on the same rough surface is reduced compared to that in a smooth surface. The central film thickness is also reduced and is smaller than that of the outlet area as the hardness of the particles is increased. Moreover, the maximum pressure of EHL increases more significantly as the particle becomes harder.

(2) The existence of hard particles increases the traction force of EHL, while that of soft particles has the opposite effect. For soft particles, when the buried depth is $D=0.2 \mathrm{~mm}$, the lubrication condition is the best. The friction interface with soft particles has better lubrication performance when the particle spacing $H_{s}<0.9 \mathrm{~mm}$, whereas the hard particle interface is favorable for EHL when $H_{s}>0.9 \mathrm{~mm}$. The particle side length has little effect on EHL.

(3) Compared with the other two rough surfaces, the square rough surface has a significant advantage for increasing the minimum thickness of EHL, and the shear force of the EHL film bearing on the square rough surface is the smallest.

(4) When the working conditions change, the soft particles have more advantages and adaptability to increase the EHL film thickness compared to the hard particles.

\section{Nomenclature}

$b$ : $\quad$ Half-width of contact area
D: Burial depth of the particles

e: Eigenstrains

$E_{d}^{\prime}$ : $\quad$ Equivalent elastic modulus of contact zone

$E_{m}$ : Young's modulus of half space

$E_{s}$ : Young's modulus of cylindrical contact

$E_{I}$ : Young's modulus of inclusion

$F_{x_{1}}$ : Friction force along $x_{1}$ direction

$h$ : $\quad$ Fluid film thickness of EHL

$H$ : Dimensionless fluid film thickness of EHL

$H_{m}$ : Minimum film thickness

$H_{c}$ : Central film thickness of the contact area

$L: \quad$ Side length of the particle

$H_{00}$ : Fluid film constant

$n_{p}$ : Number of the buried particles

$p: \quad$ Fluid pressure

$p_{H}$ : Maximum value of Hertz pressure

$P: \quad$ Dimensionless fluid pressure

$P_{\mathrm{cm}}$ : Change magnitude of the pressure

$P_{m}$ : Maximum pressure

Re: Equivalent curvature radius of up and down surface

$u_{I}: \quad$ Surface displacement caused by particles

$U_{I}$ : Dimensionless surface displacement caused by particles

$u_{r x_{1}}$ : Relative velocity of up and down surface along $x_{1}$ direction

$u_{1}$ : Speed of the half space in $x_{1}$ direction

$u_{2}$ : Speed of cylindrical contact in $x_{1}$ direction

$u_{s}: \quad$ Average velocity

$v_{1}$, Poisson's ratios of half-space and cylindrical contact,

$\nu_{2}$ : respectively

$\omega$ : $\quad$ Applied load

$\lambda, \mu$ : Lamé constants of matrix

$\alpha: \quad$ Pressure-viscosity coefficient

$\phi: \quad$ Newtonian potential, $\phi=1 / R$

$\eta$ : $\quad$ Viscosity

$H_{d}$ : Particle spacing along the $X_{1}$ direction

$V_{d}$ : Particle spacing along the $X_{2}$ direction.

\section{Data Availability}

The raw data from all simulation (UI, P, H, Rs) used to support the findings of this study are available from the corresponding author upon request.

\section{Disclosure}

The authors would like to inform that a small part of their simulation results were previously published in a poster form.

\section{Conflicts of Interest}

The authors declare no conflicts of interest regarding the publication of this paper.

\section{Acknowledgments}

The project was supported in part by the National Natural Science Foundation of China (nos. 51875417 and 51705377) for financial support. The authors also thank the Congchang 
Zhan Hydraulic Team of Wuhan University of Science and Technology for allowing presentation of a part of simulation results at their 2017 academic research poster.

\section{References}

[1] T. Mura, "Micromechanics of defects in solids," Mechanics of Elastic and Inelastic Solids, vol. 3, no. 2, pp. 177-239, 1987.

[2] J. D. Eshelby, "The determination of the elastic field of an ellipsoidal inclusion, and related problems," Proceedings of the Royal Society A: Mathematical, Physical and Engineering Sciences, vol. 241, no. 1226, pp. 376-396, 1957.

[3] K. Zhou, W. Wayne Chen, L. M. Ai et al., "Multiple 3D inhomogeneous inclusions in a half space under contact loading," Mechanics of Materials, vol. 43, no. 8, pp. 444-457, 2011.

[4] K. E. Koumi, L. Zhao, J. Leroux, T. Chaise, and D. Nelias, "Contact analysis in the presence of an ellipsoidal inhomogeneity within a half space," International Journal of Solids and Structures, vol. 51, no. 6, pp. 1390-1402, 2014.

[5] Q. Zhou, X. Jin, Z. Wang, J. Wang, L. M. Keer, and Q. Wang, "Numerical EIM with 3D FFT for the contact with a smooth or rough surface involving complicated and distributed inhomogeneities," Tribology International, vol. 93, pp. 91-103, 2016.

[6] G. Zuccaro, S. Trotta, S. Sessa, F. Marmo, and L. Rosati, "Analytical solution of elastic fields induced by a 2D inclusion of arbitrary polygonal shape," Procedia Structural Integrity, vol. 6, pp. 236-243, 2017.

[7] Z. Wang, D. Zhu, and Q. Wang, "Elastohydrodynamic lubrication of inhomogeneous materials using the equivalent inclusion method," Journal of Tribology, vol. 136, no. 2, Article ID 021501, 2013.

[8] Z. Wang, C. Yu, and Q. Wang, "Model for elastohydrodynamic lubrication of multilayered materials," Journal of Tribology, vol. 137, no. 1, Article ID 011501, 2014.

[9] Z. Shengguang, W. Wenzhong, and Z. Ziqiang, "Elastohydrodynamic lubrication analysis of point contacts with consideration of material inhomogeneity," Journal of Tribology, vol. 136, no. 4, Article ID 041501, 2014.

[10] Q. Dong and K. Zhou, "Modeling heterogeneous materials with multiple inclusions under mixed lubrication contact," International Journal of Mechanical Sciences, vol. 103, pp. 89-96, 2015.

[11] B. Zhao, B. Zhang, and K. Zhang, "Modelling three-dimensional soft elastohydrodynamic lubrication contact of heterogeneous materials," Tribology International, vol. 129, pp. 377-389, 2019.

[12] K. Chen, L. Zeng, Z. Wu, and F. Zheng, "Elastohydrodynamic lubrication in point contact on the surfaces of particlereinforced composite," AIP Advances, vol. 8, no. 4, p. 045213, 2018.

[13] N. Patir and H. S. Cheng, "An average flow model for determining effects of three-dimensional roughness on partial hydrodynamic lubrication," Journal of Lubrication Technology, vol. 100, no. 1, pp. 12-17, 1978.

[14] T. E. Tallian, "On competing failure modes in rolling contact," A S L E Transactions, vol. 10, no. 4, pp. 418-439, 1967.

[15] T. E. Tallian, "Pressure and traction rippling in elastohydrodynamic contact of rough surfaces," Journal of Lubrication Technology, vol. 96, no. 3, pp. 398-406, 1974.

[16] D. Zhu, H. S. Cheng, and B. J. Hamrock, "Effect of surface roughness on pressure spike and film constriction in elastohydrodynamically lubricated line contacts," Tribology Transactions, vol. 33, no. 2, pp. 267-273, 1990.

[17] I. Krupka, P. Sperka, and M. Hartl, "Effect of surface roughness on lubricant film breakdown and transition from EHL to mixed lubrication," Tribology International, vol. 100, pp. 116-125, 2016.

[18] J. Guegan, A. Kadiric, and H. Spikes, "A study of the lubrication of EHL point contact in the presence of longitudinal roughness," Tribology Letters, vol. 59, no. 1, 2015.

[19] S. Akbarzadeh and M. M. Khonsari, "Thermoelastohydrodynamic analysis of spur gears with consideration of surface roughness," Tribology Letters, vol. 32, no. 2, pp. 129-141, 2008.

[20] C. J. A. Roelands, "Correlational aspects of the viscositytemperature-pressure relationship of lubricating oils," Journal of Lubrication Technology, vol. 93, no. 1, p. 209, 1966.

[21] L. Houpert, "New results of traction force calculations in elastohydrodynamic contacts," Journal of Tribology, vol. 107, no. 2, pp. 241-245, 1985. 